Research Article

\title{
Clinical Relevance and Antimicrobial Profiling of Methicillin-Resistant Staphylococcus aureus (MRSA) on Routine Antibiotics and Ethanol Extract of Mango Kernel (Mangifera indica L.)
}

\author{
Ali Al Bshabshe $\mathbb{D}^{1},{ }^{1}$ Martin R. P. Joseph, ${ }^{2}$ Amgad A. Awad El-Gied, ${ }^{3}$ Abdalla N. Fadul, ${ }^{4}$ \\ Harish C. Chandramoorthy $\mathbb{D}^{2}{ }^{2}$ and Mohamed E. Hamid $\mathbb{D}^{2}$ \\ ${ }^{1}$ Department of Internal Medicine, College of Medicine, King Khalid University, Abha, Saudi Arabia \\ ${ }^{2}$ Department of Microbiology and Clinical Parasitology, College of Medicine, King Khalid University, Abha, Saudi Arabia \\ ${ }^{3}$ Department of Pharmaceutics, College of Pharmacy, King Khalid University, Abha, Saudi Arabia \\ ${ }^{4}$ Department of Clinical Laboratory Sciences, College of Applied Medical Sciences, King Khalid University, Abha, Saudi Arabia
}

Correspondence should be addressed to Harish C. Chandramoorthy; ccharishjabali@gmail.com and Mohamed E. Hamid; mehamid3@gmail.com

Received 3 October 2019; Revised 25 December 2019; Accepted 31 December 2019; Published 19 February 2020

Academic Editor: Abdelwahab Omri

Copyright (c) 2020 Ali Al Bshabshe et al. This is an open access article distributed under the Creative Commons Attribution License, which permits unrestricted use, distribution, and reproduction in any medium, provided the original work is properly cited.

\begin{abstract}
Methicillin-resistant Staphylococcus aureus (MRSA) is known for serious health problems. Testing new inexpensive natural products such as mango kernel (Mangifera indica L., Anacardiaceae) may provide alternative and economically viable anti-MRSA drugs. In the current study, we screened clinical isolates from Aseer Central Hospital, Saudi Arabia, during 2012-2017 for MRSA and tested an ethanolic extract of mango kernel for anti-MRSA activity. Brief confirmation of MRSA was performed by the Vitek 2 system, while antibiotic sensitivity of strains was tested for their clinical relevance. The In vitro disc diffusion method was used to test the anti-MRSA activity of the ethanolic mango kernel extract. The antimicrobial activity of mango kernel was compared to that of standard drugs (oxacillin and vancomycin). Of the identified $132 \mathrm{~S}$. aureus strains, 42 (31.8\%) were found to be MRSA and their prevalence showed a clear increase during the last two years $(2016-2017 ; p<0.001)$. MRSA strains showed $100 \%$ sensitivity to vancomycin, teicoplanin, linezolid, tetracycline, daptomycin, tigecycline, and tobramycin and $100 \%$ resistance to ampicillin and $98 \%$ to penicillin. The ethanolic extracts of mango kernel were found active against both $S$. aureus and the MRSA strains. Inhibitory activities (mean $\pm \mathrm{SE}$ ) were achieved at concentrations of $50 \mathrm{mg} / \mathrm{mL}(20.77 \pm 0.61), 5 \mathrm{mg} / \mathrm{mL}(16.18 \pm 0.34)$, and $0.5 \mathrm{mg} /$ $\mathrm{mL}(8.39 \pm 0.33)$ exceeding that of vancomycin $(p=0.0162)$. MRSA strains were sensitive to mango kernel extracts when compared to vancomycin. Therefore, ethanolic extracts of mango kernel can be escalated to animal model studies as a promising leading anti-MRSA drug candidate and can be an economic alternative to high-priced synthetic antibiotics.
\end{abstract}

\section{Introduction}

Staphylococcus aureus is a Gram-positive, ubiquitous bacterial pathogen that can perfectly adapt and is capable of living in different states. It has been reported that S. aureus can survive in an inanimate environment, existing as a colonizer or commensal, and may form biofilms [1]. S. aureus is an important cause of a wide range of clinical infections including bacteraemia and infective endocarditis, osteoarticular, skin and soft tissue, pleuropulmonary, and device-related infections [2]. The spread of methicillin-resistant $S$. aureus (MRSA), especially in nosocomial health settings, is a well-known health threat and economic burden worldwide [3].

The introduction of newer potent systemic antibiotic combinations failed to control the endemic reservoir of 
multidrug-resistant bacteria and suggests that such policies have little impact [4]. There are reports of increasing drug resistance against human pathogens as well as undesirable side effects of certain antimicrobial agents. The overall global trend shows alarming rise and expansion of MRSA [5]. A meta-analysis showed an obvious connection between exposure to antibiotics and MRSA prevalence, a crucial information which may be considered when planning future MRSA control policies [6]. The literature demonstrates a clinically important burden of $S$. aureus related to various types of infection operations and a substantial contribution of MRSA. Preventive strategies aimed specifically at MRSA could reduce healthcare costs and improve patient outcomes $[7,8]$. The prevalence of MRSA is high and rapidly increasing worldwide but is a major public health worry in developing countries. Lack of surveys and collection of epidemiological data and absence of efficient health control programs are contributing to the spread of MRSA [9].

It is necessary to search for new agents that are better, cheaper, and without side effect for treating infectious diseases especially in developing countries. It has been long known that an extensive variety of plant and plant-derived natural products are used in the treatment of infections. Phytoconstituents have been found to inhibit bacteria, fungi, viruses, and pests [10]. The curative effect and appropriateness of products derived from natural plants against diseases are a recognized and documented reality. Mangifera indica (mango kernel) is an example which was recorded to have antimicrobial activities. Application of such innovative results may help in controlling the infection and preventing their spread.

Previous studies have shown that extracts of mango kernel are promising candidates against numerous species of pathogenic bacteria [11-13]. Leaves of mango kernel have been reported to possess antibacterial activity against Escherichia coli including members of Enterobacteriaceae family, while mangiferin, a bioactive component, has been reported to possess remarkable anti-influenza activity [14]. Both acetone and methanol extracts inhibited the growth of Gram-positive bacteria, with a zone of inhibition between 15 and $16 \mathrm{~mm}$ and a zone of $14 \mathrm{~mm}$ to Gram-negative bacterium Salmonella typhi at $250 \mathrm{mg} / \mathrm{mL}$. Thai mango (M. indica L. cv. "Fahlun") seed kernel extract was found to have potent antimicrobial properties [15]. Aqueous and chloroform extracts of the leaves and stem of mango kernel were found to be active as an antibacterial agent against S. Aureus [16]. Mangiferin was found to have antibacterial activity in vivo against specific periodontal pathogens such as Actinobacillus actinomycetemcomitans, Prevotella intermedia, Porphyromonas gingivalis, Fusobacterium nucleatum, and Peptostreptococcus sp. It has been suggested to be used as an alternative remedial agent or an adjunctive treatment along with classic antibiotics $[15,17]$.

This study aimed to examine the clinical significance of MRSA and to determine their antimicrobial profile against routine antimicrobial agents and against ethanolic extracts of mango kernel.

\section{Materials and Methods}

2.1. Study Design. This study was conducted between 2012 and 2017 at definite time intervals and duration at the Department of Microbiology and Clinical Parasitology, College of Medicine, King Khalid University, and Aseer Central Hospital, Abha, Saudi Arabia. Ethical approval was obtained from the Ethics Committee of the College of Medicine, King Khalid University, vide reference letter, REC \# 2017-02-17. Patient information, clinical data, and bacteriological and antimicrobial records of the isolates were collected and saved in a spreadsheet for analysis.

2.2. Inclusion and Exclusion Criteria. All isolates were Grampositive coccus and catalase- and coagulase-positive. Coagulase-negative staphylococci and duplicate isolates were excluded. Both MRSA and other coagulase-positive $S$. aureus strains were included in the study for comparative reasons.

2.3. Bacterial Isolates. S. aureus strains $(n=132)$ were isolated from various clinical samples of patients attending Aseer Central Hospital, Abha, Saudi Arabia. Samples were processed for culture by standard conventional methods, and initial identification was performed by standard phenotypic tests [18, 19]. Isolates that were Gram-positive coccus and catalase- and coagulase-positive were collected. Subsequent initial bench identification confirmation of MRSA was done at the microbiology laboratory using the Vitek 2 identification system (BioMerieux, France) according to the manufacture standards.

2.4. Antibiotic Susceptibility Testing (AST). Antimicrobial susceptibility testing was carried out using the Vitek 2 system, according to the manufacturer's information. The following antimicrobial agents have been tested for at least one or more strains: Amikacin; Amox/K Clav; Amp/Subactm; Ampicillin; Azithromycin; Cefepime; Cefotaxime; Cefoxitin; Cefuroxime; Cephalothin; Chloramphenicol; Ciprofloxacin; Clarithromycin; Clindamycin; Colistin; Cotrimox; Daptomycin; Erythromycin; Fosfomycin; Fusidc Acid; Gentamicin; Imipenem; Levofloxacin; Linezolid; Meropenem; Moxifloxacin; Mupirocin; Nalidixic; Neomycin; Nitrofurantoin; Norfloxacin; Ofloxacin; Oxacill/ Methicill; Oxacillin; Penicillin; Pip/Tazo; Piperacillin; Rifampin; Sulfamethoxazole; Synercid; Teicoplanin; Tetracyclin; Tigecycline; Tobramycin; Trimeth/Sulfa; and Vancomycin.

2.5. Oxacillin Screen. Oxacillin screen agar testing was performed for $S$. aureus according to the NCCLS methodology [20] using Mueller-Hinton agar with $4 \% \mathrm{NaCl}$ and oxacillin $(6 \mu \mathrm{g} / \mathrm{ml})$. Plates were inoculated with the 0.5 $\mathrm{McFarland}$ bacterial suspensions and incubated at $35^{\circ} \mathrm{C}$ for $24 \mathrm{~h}$. 
2.6. Plant Collection and Identification. Fresh mango kernel was collected from local markets in Abha, Saudi Arabia and was identified and authenticated at the Department of Biological Sciences, College of Science, King Khalid University. The collected seeds were stored in polythene bags at room temperature till further use.

2.7. Extraction of Plant Seeds. Mango kernel seeds were cleaned, air-dried, and coarsely powdered. 1000 grams of powder was extracted with ethanol in a Soxhlet apparatus. The extracts were filtered, and the filtrates were vaporized to dryness and weighed in order to determine the \% yield of the extracts, following the formula: $\%$ yield $=$ (weight of extract/weight of ground plant material $) \times 100$. The stock solutions of the crude ethanolic extracts were prepared by dilution of the dried extracts with $50 \%$ ethanol to obtain the desired final concentrations of $50 \mathrm{mg} / \mathrm{mL}, 5 \mathrm{mg} /$ $\mathrm{mL}, 0.5 \mathrm{mg} / \mathrm{mL}$, and $0.05 \mathrm{mg} / \mathrm{mL}$. These concentrations were used to impregnate filter paper disks $(5.5 \mathrm{~mm}$ diameters).

Disks impregnated into $50 \%$ ethanol were used as control, while standard antimicrobial disks such as oxacillin and vancomycin (Difco) were used as positive control.

2.8. Antimicrobial Assays of Plant Extracts against MRSA. MRSA bacterial strains $(n=51)$ recovered from clinical samples isolated from patients at Aseer Central Hospital were used in the study. One to three loopful of $24 \mathrm{~h}$ old cultures from each test strains were used to prepare 0.5 McFarland standard suspensions.

2.9. Determination of $M I C$ and $M B C$ Values. Minimum inhibitory concentration (MIC) of ethanolic extraction of mango kernel was determined by using descending concentrations of the extract. The MIC of the extract was diluted using sterile saline and was tested for antibacterial activity against Staphylococcus aureus and MRSA according to [21] with some modifications. The different prepared concentrations were tested against the bacterial strains using disc diffusion assay as previously mentioned. The formed clear zones were measured and recorded. Serial dilution was determined starting with a concentration of $1 \mathrm{mg} / \mathrm{mL}$ in the tubes containing $2 \mathrm{~mL}$ of sterile Mueller-Hinton broth and discarded $2 \mathrm{~mL}$ from the final tube. One tube with Mueller-Hinton broth as positive control and one tube with Mueller-Hinton broth as negative control were used for the test. $0.2 \mathrm{~mL}$ of bacterial suspension ranging from 0.5 to $1 \mathrm{Mcfarland}$ was added to all tubes except to the negative control tube, and all the tubes were incubated overnight at $37^{\circ} \mathrm{C}$ aerobically. Duplication of each MIC test was performed. After the overnight incubation, subculture of each tubes was prepared by the conventional plating method on Mueller-Hinton agar to examine the growth or no growth, and MIC of the test was calculated as the mean of lowest concentration of inhibition and the highest concentration allows the growth of the test microorganism.

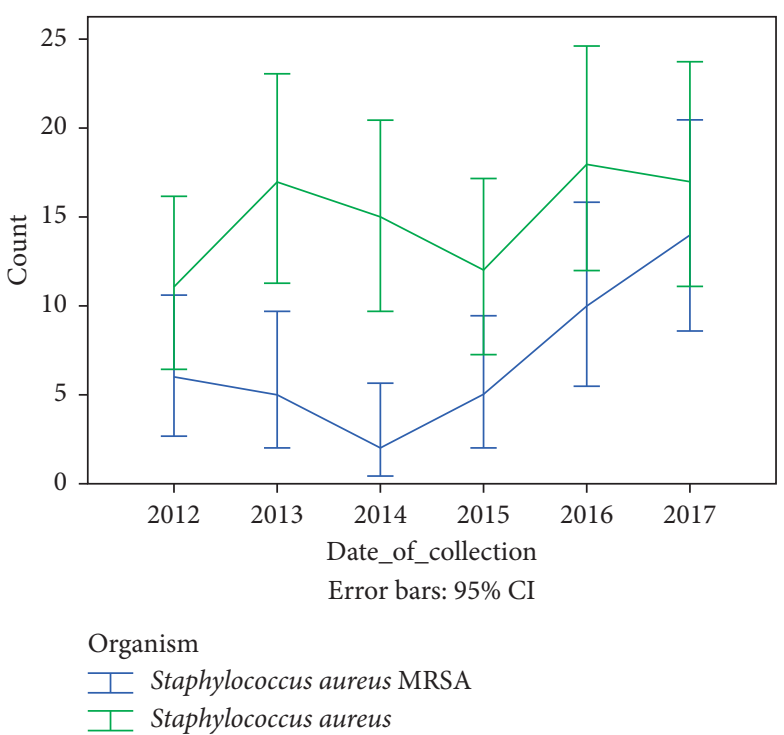

FIGURE 1: Prevalence of MRSA and other S. aureus strains isolated from various clinical samples of patients attending Aseer Central Hospital, Abha, Saudi Arabia in five years (2016-2017).

2.10. Statistical Analysis. Statistical analysis of data obtained from triplicate experiments was performed using SPSS software version 16.0. The nonparametric test for several independent samples was used to compare variation among means of different inhibition zones induced by extracts in comparison with positive controls. Linear regression was applied to assess the relationships among several independent samples. $p$ value $<0.05$ was considered as the level of significance.

\section{Results}

3.1. Prevalence of MRSA and S. aureus Strains. Prevalence of MRSA and other $S$. aureus strains isolated from various clinical materials of patients attending Aseer Central Hospital, Abha, Saudi Arabia in five years (2016-2017) is shown in Figure 1. Of the 132 suspected S. aureus strains, 42 (31.8\%) were found to be MRSA and the rest (68.2\%) were S. aureus. The prevalence of MRSA demonstrated an obvious rise during 2016-2017 compared to the previous three years $(p<0.001)$.

3.2. Distribution of MRSA and S. aureus Strains according to Clinical Specimens and Age Groups. MRSA and other S. aureus strains originated from all age groups, but the majority was from 20 - to 40 -year-old patients. The strains were recovered predominantly from the respiratory specimens (77.3\%) including endotracheal tube (53.8\%), throat swab $(10.6 \%)$, sputum $(6.8 \%)$, and tracheal secretion (5.3\%), followed by blood (10.6\%) and wound specimens (4.5\%).

3.3. Susceptibility of MRSA and S. aureus Strains to Routine Antimicrobials. The susceptibility of MRSA and S. aureus strains to routine antimicrobials is shown in Figure 2. MRSA strains showed $100 \%$ sensitivity to vancomycin, teicopanin, 


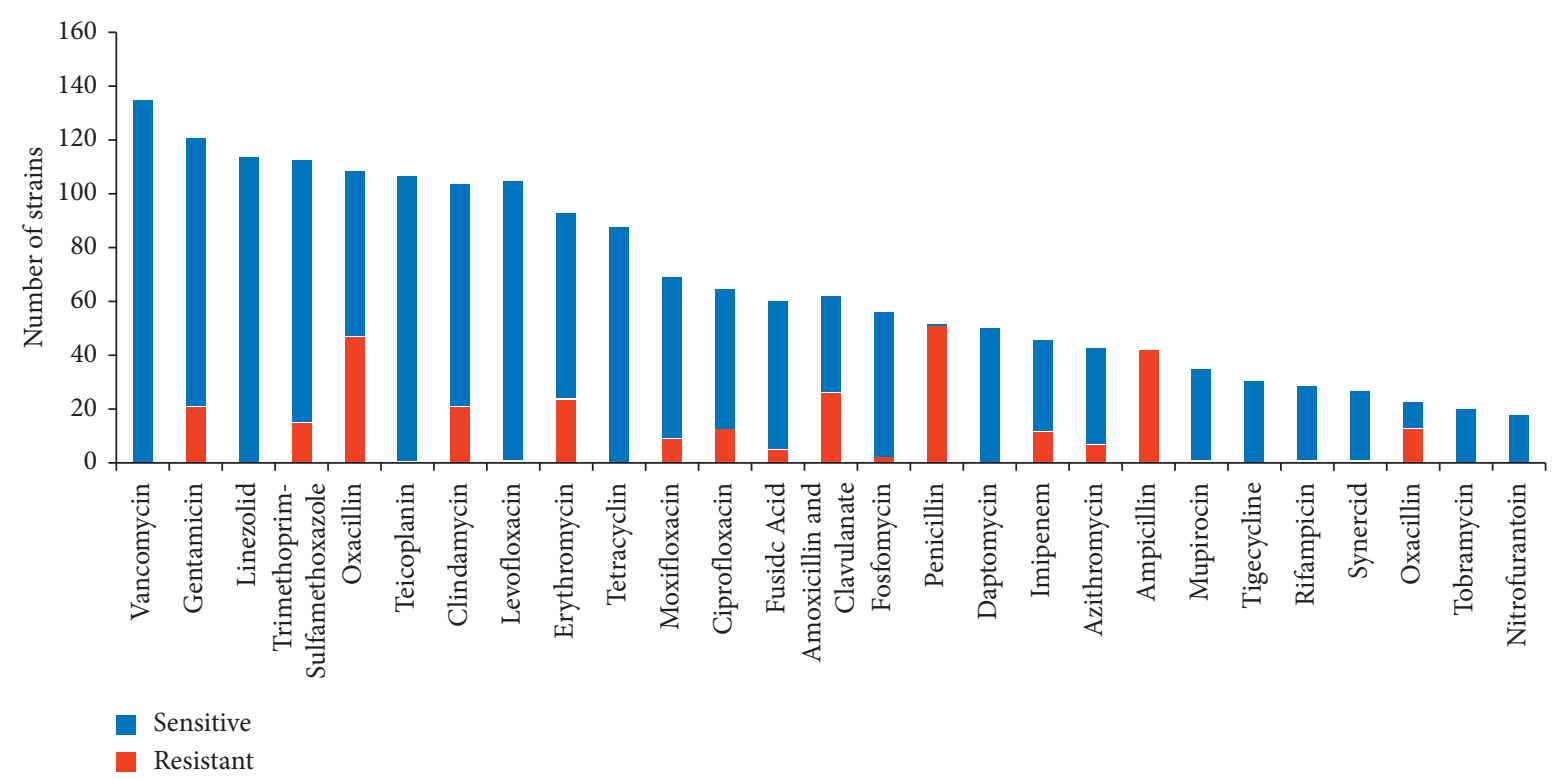

(a)

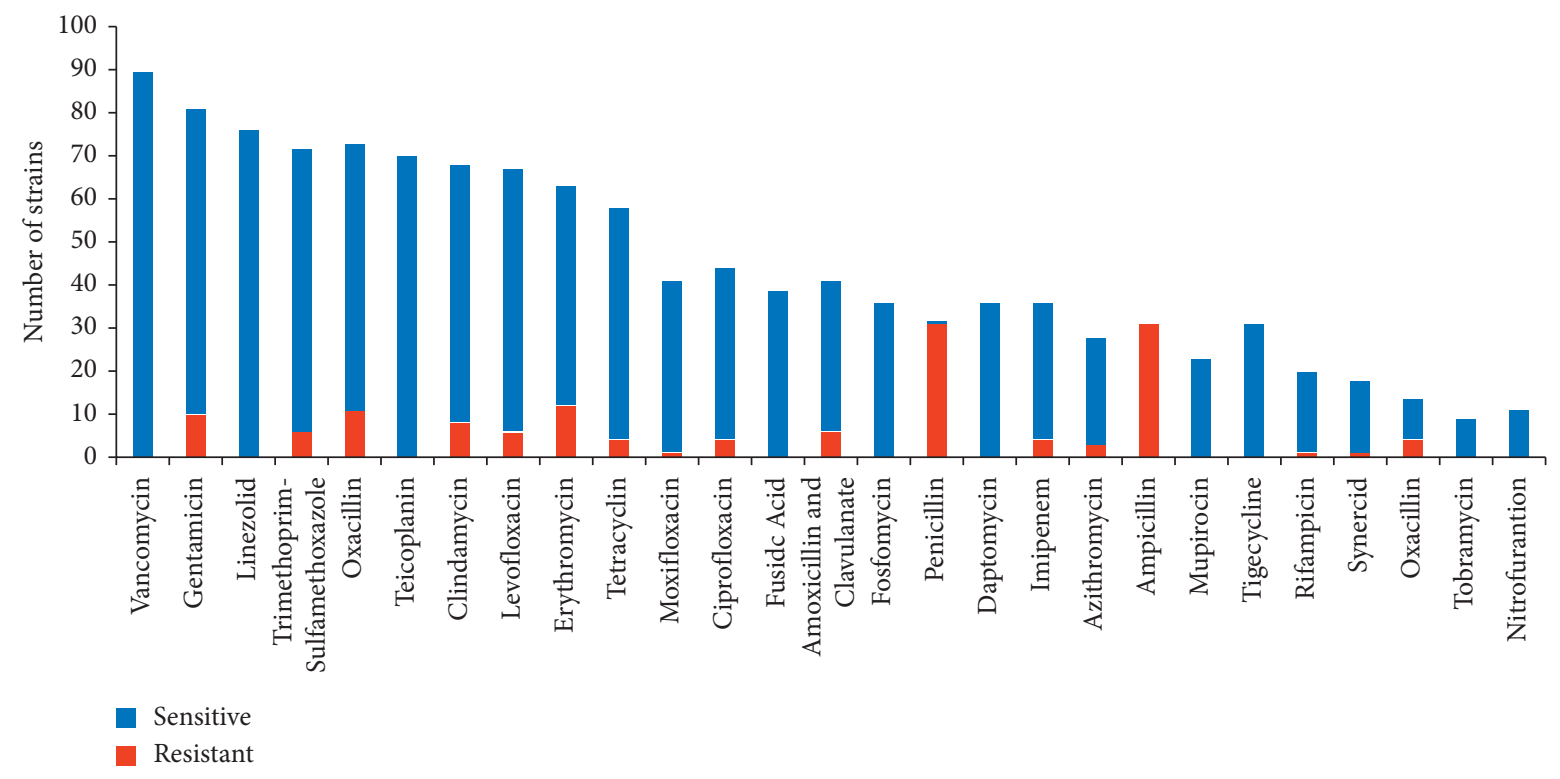

(b)

Figure 2: Susceptibility of MRSA and S. aureus strains to routine antimicrobials. Strains were isolated from various clinical samples of patients attending Aseer Central Hospital, Abha, Saudi Arabia, in five years (2016-2017). (a) Methicillin-resistant Staphylococcus aureus (MRSA). (b) Methicillin-sensitive Staphylococcus aureus (MRSA).

linezolid, tetracycline, daptomycin, tigecycline, and tobramycin and more than $99 \%$ sensitivity to teicoplanin and levofloxacin. On the other hand, MRSA strains showed $100 \%$ resistance to ampicillin and $98 \%$ resistance to penicillin. The ethanolic extracts of mango kernel were found to be active against both $S$. aureus and the MRSA strains. Inhibitory activities (mean $\pm \mathrm{SE}$ ) were achieved at concentrations of $50 \mathrm{mg} / \mathrm{mL}(20.77 \pm 0.61), 5 \mathrm{mg} / \mathrm{mL}(16.18 \pm 0.34)$, and $0.5 \mathrm{mg} / \mathrm{mL} \quad(8.39 \pm 0.33)$ but not at $0.05 \mathrm{mg} / \mathrm{mL}$ $(0 \pm 0.00)$. Inhibition zone by oxacillin was $9.45 \mathrm{~mm}$ while vancomycin was $20 \pm 0.24 \mathrm{~mm}$. The MIC was found $(0.15625 \mathrm{mg} / \mathrm{mL})$, whereas the $\mathrm{MBC}$ was $(0.25 \mathrm{mg} / \mathrm{mL})$. The antagonistic value of mango kernel at $50 \mathrm{mg} / \mathrm{mL}$ exceeded that of vancomycin ( $p=0.0162$ ) but at $5 \mathrm{mg} / \mathrm{mL}$ was found to be less active than vancomycin $(p<0.001)$; nevertheless, at this concentration it exceeded oxacillin $(p<0.001)$.

\subsection{Susceptibility of MRSA and S. aureus Strains to Various} Concentrations of Ethanolic Extract of Mango Kernel. The susceptibility of MRSA and S. aureus strains to various concentrations of ethanolic extract of mango kernel is shown in Table 1.

The ethanolic extracts of mango kernel were found active against both $S$. aureus and the MRSA strains (Figure 3). Inhibitory activities (mean $\pm \mathrm{SE}$ ) were achieved at 
TABLE 1: Susceptibility of MRSA and S. aureus to various concentrations of ethanolic extract of mango kernel.

\begin{tabular}{|c|c|c|c|c|c|c|c|c|c|}
\hline \multirow{3}{*}{ SN } & \multirow{3}{*}{ Strain } & \multirow{3}{*}{ Source } & \multicolumn{7}{|c|}{ Inhibition (mm) } \\
\hline & & & \multicolumn{4}{|c|}{ M. indica } & \multirow{2}{*}{$\begin{array}{l}\text { Oxacillin } \\
\text { (mm) } \\
1 \mu \mathrm{g}\end{array}$} & \multirow{2}{*}{$\begin{array}{l}\text { Vancomycin } \\
(\mathrm{mm}) \\
30 \mu \mathrm{g}\end{array}$} & \multirow[b]{2}{*}{$\begin{array}{c}\text { Negative control } \\
\text { (ethanol-impregnated } \\
\text { discs) }\end{array}$} \\
\hline & & & $50 \mathrm{mg} / \mathrm{mL}$ & $5 \mathrm{mg} / \mathrm{mL}$ & $0.5 \mathrm{mg} / \mathrm{mL}$ & $0.05 \mathrm{mg} / \mathrm{mL}$ & & & \\
\hline 1 & S. aureus & ATCC 25923* & 21 & 14 & 9 & 0 & 23 & 20 & 0 \\
\hline 2 & MRSA & Strain $367^{* *}$ & 25 & 15 & 7 & 0 & 7 & 19 & 0 \\
\hline 3 & MRSA & Strain 1652 & 20 & 14 & 6 & 0 & 9 & 21 & 0 \\
\hline 4 & MRSA & Strain R68 & 24 & 17 & 7 & 0 & 8 & 22 & 0 \\
\hline 5 & MRSA & Strain 1205 & 24 & 18 & 9 & 0 & 8 & 18 & 0 \\
\hline 6 & MRSA & Strain 300 & 24 & 19 & 10 & 0 & 8 & 20 & 0 \\
\hline 7 & MRSA & Strain 301 & 24 & 18 & 10 & 0 & 9 & 19 & 0 \\
\hline 8 & MRSA & Strain 475 & 23 & 17 & 10 & 0 & 8 & 21 & 0 \\
\hline 9 & MRSA & Strain 2 & 20 & 18 & 8 & 0 & 8 & 22 & 0 \\
\hline 10 & MRSA & Strain CL2 & 20 & 17 & 8 & 0 & 9 & 18 & 0 \\
\hline 11 & MRSA & Strain 396 & 19 & 18 & 8 & 0 & 10 & 20 & 0 \\
\hline 12 & MRSA & Strain 682 & 20 & 18 & 9 & 0 & 7 & 19 & 0 \\
\hline 13 & MRSA & Strain 6821 & 23 & 19 & 8 & 0 & 5 & 21 & 0 \\
\hline 14 & S. aureus & Strain 1946 & 19 & 17 & 13 & 0 & 17 & 22 & 0 \\
\hline 15 & MRSA & Strain 1947 & 20 & 16 & 11 & 0 & 16 & 18 & 0 \\
\hline 16 & MRSA & Strain 1533 & 25 & 20 & 13 & 0 & 0 & 20 & 0 \\
\hline 17 & MRSA & Strain 634 & 20 & 14 & 12 & 0 & 8 & 19 & 0 \\
\hline 18 & S. aureus & Strain 3966 & 20 & 14 & 12 & 0 & 16 & 21 & 0 \\
\hline 19 & MRSA & Strain 491 & 19 & 17 & 10 & 0 & 8 & 22 & 0 \\
\hline 20 & MRSA & Strain S77 & 20 & 12 & 8 & 0 & 5 & 18 & 0 \\
\hline 21 & S. aureus & Strain 528 & 23 & 18 & 12 & 0 & 25 & 20 & 0 \\
\hline 22 & MRSA & Strain CL1 & 20 & 17 & 6 & 0 & 10 & 19 & 0 \\
\hline 23 & MRSA & Strain E2 & 20 & 18 & 14 & 0 & 9 & 21 & 0 \\
\hline 24 & MRSA & Strain 19657 & 21 & 19 & 8 & 0 & 4 & 22 & 0 \\
\hline 25 & MRSA & Strain 688 & 17 & 15 & 7 & 0 & 10 & 18 & 0 \\
\hline 26 & MRSA & Strain 677 & 20 & 17 & 8 & 0 & 10 & 20 & 0 \\
\hline 27 & MRSA & Strain 475 & 23 & 19 & 8 & 0 & 8 & 19 & 0 \\
\hline 28 & S. aureus & Strain 1890 & 19 & 17 & 8 & 0 & 15 & 21 & 0 \\
\hline 29 & S. aureus & Strain 3889 & 18 & 14 & 5 & 0 & 22 & 22 & 0 \\
\hline 30 & MRSA & Strain 9728 & 20 & 15 & 5 & 0 & 8 & 18 & 0 \\
\hline 31 & MRSA & Strain 715 & 20 & 17 & 9 & 0 & 7 & 20 & 0 \\
\hline 32 & MRSA & Strain 369 & 20 & 18 & 10 & 0 & 7 & 19 & 0 \\
\hline 33 & MRSA & Strain 319 & 21 & 12 & 4 & 0 & 5 & 21 & 0 \\
\hline 34 & MRSA & Strain S-66 & 20 & 17 & 7 & 0 & 8 & 22 & 0 \\
\hline 35 & MRSA & Strain S-85 & 21 & 19 & 7 & 0 & 7 & 18 & 0 \\
\hline 36 & S. aureus & Strain 1562 & 20 & 17 & 5 & 0 & 15 & 20 & 0 \\
\hline 37 & S. aureus & Strain 109 & 20 & 15 & 8 & 0 & 17 & 19 & 0 \\
\hline 38 & MRSA & Strain 394 & 20 & 16 & 10 & 0 & 7 & 21 & 0 \\
\hline 39 & MRSA & Strain 3941 & 20 & 17 & 11 & 0 & 8 & 22 & 0 \\
\hline 40 & MRSA & Strain 3677 & 22 & 15 & 7 & 0 & 7 & 18 & 0 \\
\hline 41 & MRSA & Strain 3678 & 21 & 15 & 8 & 0 & 7 & 20 & 0 \\
\hline 42 & MRSA & Strain 3679 & 20 & 14 & 7 & 0 & 8 & 19 & 0 \\
\hline 43 & MRSA & Strain 3680 & 20 & 15 & 7 & 0 & 8 & 21 & 0 \\
\hline 44 & MRSA & Strain 3689 & 21 & 15 & 8 & 0 & 7 & 22 & 0 \\
\hline 45 & MRSA & Strain 3687 & 20 & 14 & 7 & 0 & 8 & 18 & 0 \\
\hline 46 & MRSA & Strain 3686 & 20 & 15 & 7 & 0 & 8 & 20 & 0 \\
\hline 47 & MRSA & Strain 3685 & 21 & 15 & 8 & 0 & 7 & 19 & 0 \\
\hline 48 & MRSA & Strain 3681 & 20 & 14 & 7 & 0 & 8 & 21 & 0 \\
\hline 49 & MRSA & Strain 3682 & 20 & 15 & 7 & 0 & 8 & 22 & 0 \\
\hline 50 & MRSA & Strain 3684 & 21 & 15 & 8 & 0 & 7 & 18 & 0 \\
\hline 51 & MRSA & Strain 3688 & 20 & 14 & 7 & 0 & 8 & 20 & 0 \\
\hline
\end{tabular}

Abbreviations: S. aureus, Staphylococcus aureus; MRSA, methicillin-resistant Staphylococcus aureus. *ATCC, American Type Culture Collection; **strains originated from clinical materials, Aseer Central Hospital, Abha, Saudi Arabia. 


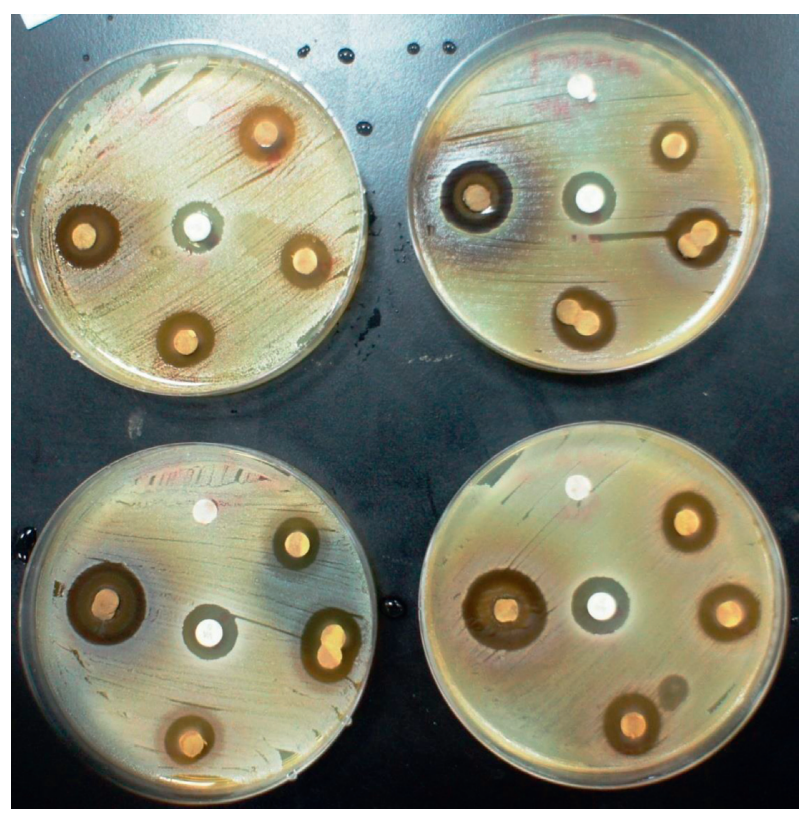

FIGURE 3: Inhibitory activities (clearance) of ethanolic extracts of mango kernel against both $S$. aureus and the MRSA strains.

concentrations $\quad 50 \mathrm{mg} / \mathrm{mL} \quad(20.77 \pm 0.61), \quad 5 \mathrm{mg} / \mathrm{mL}$ $(16.18 \pm 0.34)$, and $0.5 \mathrm{mg} / \mathrm{mL}(8.39 \pm 0.33)$ but not at $0.05 \mathrm{mg} / \mathrm{mL}(0 \pm 0.00)$. The inhibition zone by oxacillin was $9.45 \mathrm{~mm}$ while the inhibition zone by vancomycin was $20 \pm 0.24 \mathrm{~mm}$. The MIC was found to be $0.15625 \mathrm{mg} / \mathrm{mL}$, whereas the $\mathrm{MBC}$ was $0.25 \mathrm{mg} / \mathrm{mL}$.

A linear regression curve illustrating the activity of various concentrations of ethanolic extract of mango kernel against $S$. aureus and MRSA is shown in Figure 4.

The antagonistic value of mango kernel at $50 \mathrm{mg} / \mathrm{mL}$ exceeded that of vancomycin $(p=0.0162)$ but at $5 \mathrm{mg} / \mathrm{mL}$, it was found to be less active than vancomycin $(p<0.001)$; nevertheless, at this concentration, it exceeded oxacillin $(p<0.001)$ (Table 2; Figure 5).

\section{Discussion}

The prevalence of MRSA is high and rapidly increasing worldwide but is a major public health worry in developing countries. MRSA is one of the important pathogens that result in heavy health and economic burden throughout the world [8]. But the actual effect of multidrug-resistant bacterial infections on real-world healthcare resources is not fully determined [22]. The spread of MRSA is becoming a well-known phenomenon and rapidly spreading worldwide. Roughly, the cost of MRSA treatment is about US8000 per case and varied in other developing countries [23].

Lack of surveys and collection of epidemiological data and absence of efficient health control programs are contributing to the spread of MRSA $[9,24]$. Though our report of MRSA is a little bit on higher side, the average data of Saudi Arabia are as comparable with the global data [25]. The results of the current study showed that testing and application innovative "safe" products like black seed or mango seeds, which have been recently recognized to have

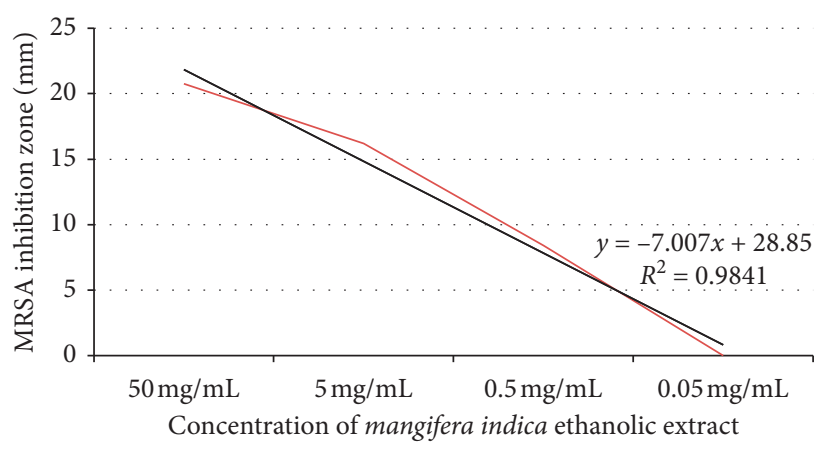

FIgURE 4: Linear regression curve illustrating activity of various concentrations of ethanolic extract of mango kernel against S. aureus and MRSA.

antimicrobial activities, can be an economic alternative to control these menacing microbes. Further this will prevent of the spread of MRSA in the hospitals and patients undergoing treatment as a priority to avoid serious consequences.

MRSA is a major contributor and huge burden in the management of nosocomial disease. The overall global trend displays a disturbing rise of MRSA infections in addition to increased occurrence of multidrug resistance (MDR) strains [5]. We had predominantly isolates from respiratory specimens which were well observed in many studies. Our observation of the about $10 \%$ from blood well conceded with studies which showed the MRSA was only next to the thirdgeneration cephalosporin-susceptible Enterobacteriaceae. However MRSA had greater impact on mortality, excess length of stay and cost more than Enterobacteriaceae infection [8]. Owing to high incidence of antibiotic-resistant microbes, natural products and herbal extracts previously reported for bioactivity have been seen as inexpensive and low or no side effects lead drugs [26]. They are therefore can be considered appropriate and economic alternative for healing drug resistant infectious diseases [27].

The antibiotic sensitivity pattern of the all the MRSA isolates were comparable thought the study period while antibiotic-resistant bacteria, including community-acquired and hospital-acquired MRSA, vancomycin-intermediate $S$. aureus, vancomycin-resistant enterococci, macrolide- and penicillin-resistant Streptococcus pneumoniae, extendspectrum beta-lactamase-producing Escherichia coli and Klebsiella pneumoniae, carbapenem-resistant Enterobacteriaceae, and multidrug-resistant Pseudomonas aeruginosa and Acinetobacter spp., are becoming prevalent in many Asian countries including Saudi Arabia [24]. Our earlier study revealed that Escherichia coli and staphylococci were the main etiological agents of infections in Aseer region, while purulent exudates of wounds and abscesses were the main source of $S$. aureus. Also, a higher rate of MRSA was detected [25].

The antimicrobial resistance among these pathogens in Saudi Arabia in the last decades and their antimicrobial resistance has increased among $S$. aureus in the Kingdom with a growing prevalence of both nosocomial and community MRSA isolates [28]. The anti-MRSA results over the clinical isolates of MRSA were promising with high 
TABLE 2: Summary of descriptive statistics of different concentrations of ethanolic extracts of mango kernel were found to be active against both $S$. aureus and the MRSA strains.

\begin{tabular}{|c|c|c|c|c|c|c|}
\hline Parameters & $50 \mathrm{mg} / \mathrm{mL}$ & $5 \mathrm{mg} / \mathrm{mL}$ & $0.5 \mathrm{mg} / \mathrm{mL}$ & $0.05 \mathrm{mg} / \mathrm{mL}$ & $1 \mu \mathrm{g}$ & $30 \mu \mathrm{g}$ \\
\hline Number of strains & 51 & 51 & 51 & 51 & 51 & 51 \\
\hline Minimum inhibition zone & 17.00 & 12.00 & 4.00 & 0.00 & 0.00 & 18.00 \\
\hline Maximum inhibition zone & 25.00 & 20.00 & 14.00 & 0.00 & 25.00 & 22.00 \\
\hline Mean inhibition zone & 20.76 & 16.18 & 8.39 & 0.00 & 9.45 & 20.00 \\
\hline Standard error & 0.24 & 0.27 & 0.30 & 0.00 & 0.67 & 0.20 \\
\hline Standard deviation & 1.73 & 1.92 & 2.17 & 0.00 & 4.75 & 1.41 \\
\hline
\end{tabular}

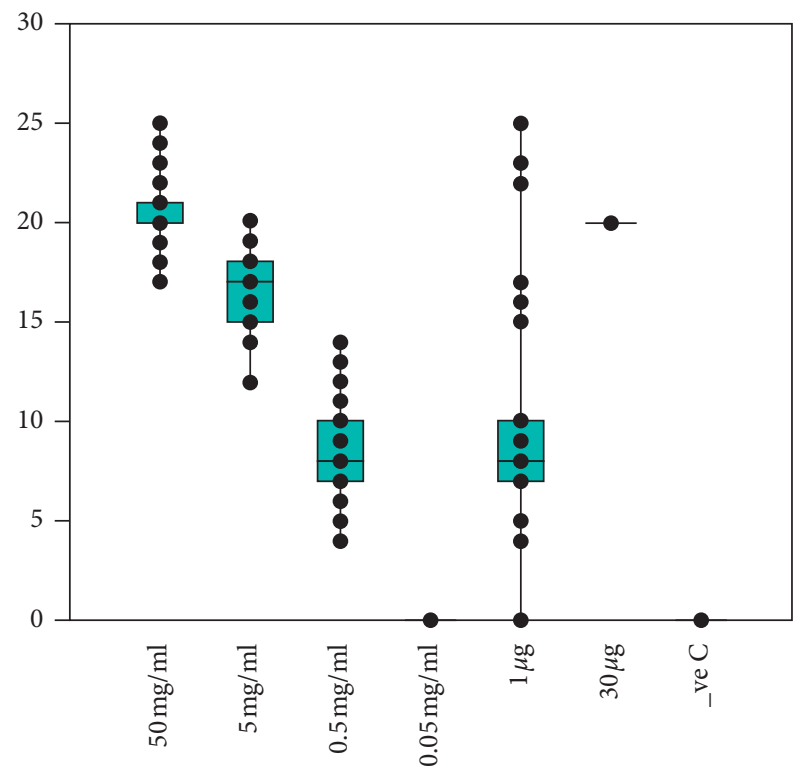

Figure 5: Antagonistic value of mango kernel at various concentrations compared to that of vancomycin and oxacillin.

discriminatory zone of inhibition between control antibiotics and extracts. The results were comparable with other similar studies done with mango kernel extracts [29]. Further based on the sensitivity pattern no vancomycinresistant MRSA have been reported, although isolates with reduced susceptibility to the drug was noted. MRSA from KSA were found linked to epidemic strains from the Middle East and possibly India, rather than to the Western European UK countries [30]. High-resolution typing methods, including SCCmec subtyping, might help to differentiate related epidemic strains and to monitor routes of transmission to identify the origin of antibiotic resistance and rational use new drugs.

\section{Conclusion}

Ethanolic extracts of mango kernel showed a significant activity against MRSA isolates. We did not observe any variation in anti-MRSA activity over five years of time nor varied isolates. Mango kernel extracts could be the best and economic alternative to combat growing antibiotic resistance observed thoughout the Middle East and Saudi Arabia. The study could be escalated to small animal models addressing the in vivo actions including toxicity, dose, and mechanism of anti-MRSA activity of mango kernel extracts.

\section{Data Availability}

The data have been incorporated in the manuscript. If further information is required, the authors will furnish the additional supporting data.

\section{Additional Points}

Highlights. The majority of MRSA strains were from the respiratory system, sensitive to vancomycin but resistant to ampicillin and penicillin. All MRSA strains were sensitive to mango kernel extract and showed in vitro susceptibility which matched that of vancomycin. This is a promising therapeutic product and can be used as an economic adjunct treatment immediately.

\section{Conflicts of Interest}

The authors declare that there are no conflicts of interest related to this study.

\section{Authors' Contributions}

M. E. H. and H. C. C. were involved in conceptualization. H. C. C., M. E. F., A. A. B., and M. R. P. were responsible for methodology. A. A. B., M. R. P., A. A. A., and A. N. F. investigated the study. M. E. H., A. A. B., and H. C. C. analysed the study. M. E. H., H. C. C., and A. A. A. provided the resources. M. E. F. and H. C. C. supervised the study. M. R. P carried out project administration. H. C. C. and M. E. H. performed funding acquisition. M. P. R., A. A. B., and M. E. F. wrote the original draft. M. E. F. and H. C. C. reviewed and edited the article. Ali Al Bshabshe and Martin R. P. Joseph equally contributed to this work.

\section{Acknowledgments}

The authors wish to thank Microbiology Departments at both Aseer Central Hospital and the College of Medicine King Khalid University for facilitating the accomplishment of the research work. The research was funded by the Deanship of Scientific Research, King Khalid University, Abha, Saudi Arabia (Project number: G.R.P 354-39).The authors wish to thank Microbiology Departments at both Aseer Central Hospital and the College of Medicine King Khalid University for facilitating the accomplishment of the research work. 


\section{References}

[1] S. Stefani and A. Goglio, "Methicillin-resistant Staphylococcus aureus: related infections and antibiotic resistance," International Journal of Infectious Diseases, vol. 14, no. Suppl 4, pp. S19-S22, 2010.

[2] S. Y. C. Tong, J. S. Davis, E. Eichenberger, T. L. Holland, and V. G. Fowler Jr., "Staphylococcus aureus infections: epidemiology, pathophysiology, clinical manifestations, and management," Clinical Microbiology Reviews, vol. 28, no. 3, pp. 603-661, 2015.

[3] M. Dadashi, M. J. Nasiri, F. Fallah et al., "Methicillin-resistant Staphylococcus aureus (MRSA) in Iran: a systematic review and meta-analysis," Journal of Global Antimicrobial Resistance, vol. 12, pp. 96-103, 2018.

[4] A. J. Petros, M. O’Connell, C. Roberts, P. Wade, and H. K. F. van Saene, "Systemic antibiotics fail to clear multidrug-resistant Klebsiella from a pediatric ICU," Chest, vol. 119, no. 3, pp. 862-866, 2001.

[5] J. Drebes, M. Kunz, C. Pereira, C. Betzel, and C. Wrenger, "MRSA infections: from classical treatment to suicide drugs," Current Medicinal Chemistry, vol. 21, no. 15, pp. 1809-1819, 2014.

[6] E. Tacconelli, G. De Angelis, M. A. Cataldo, E. Pozzi, and R. Cauda, "Does antibiotic exposure increase the risk of methicillin-resistant Staphylococcus aureus (MRSA) isolation? A systematic review and meta-analysis," The Journal of Antimicrobial Chemotherapy, vol. 61, no. 1, pp. 26-38, 2007.

[7] J. P. Sutton and C. A. Steiner, "Hospital-, health care-, and community-acquired MRSA: estimates from California hospitals: 2013: statistical brief \#212," Healthcare Cost and Utilization Project (HCUP) Statistical Briefs, Agency for Healthcare Research and Quality, Rockville, MD, USA, 2006.

[8] A. J. Stewardson, A. Allignol, J. Beyersmann et al., "The health and economic burden of bloodstream infections caused by antimicrobial-susceptible and non-susceptible Enterobacteriaceae and Staphylococcus aureus in European hospitals, 2010 and 2011: a multicentre retrospective cohort study," Eurosurveillance, vol. 21, no. 33, 2016.

[9] S. Eshetie, F. Tarekegn, F. Moges, A. Amsalu, W. Birhan, and K. Huruy, "Methicillin resistant Staphylococcus aureus in Ethiopia: a meta-analysis," BMC Infectious Diseases, vol. 16, no. 1, p. 689, 2016

[10] M. C. Marjorie, "Plant products as antimicrobial agents," Clinical and Microbiology Reviews, vol. 12, no. 4, pp. 564-582, 1999.

[11] C. S. de Oliveira, V. S. Falcão-Silva, J. P. Siqueira Jr. et al., "Drug resistance modulation in Staphylococcus aureus, a new biological activity for mesoionic hydrochloride compounds," Molecules (Basel, Switzerland), vol. 16, no. 16, pp. 2023-2031, 2011.

[12] A. A. Awad El-Gied, M. R. P. Joseph, I. M. Mahmoud, A. M. Abdelkareem, A. M. Al Hakami, and M. E. Hamid, "Antimicrobial activities of seed extracts of mango (Mangifera indica L.)," Advances in Microbiology, vol. 2, pp. 571-576, 2012.

[13] A. Hannan, S. Asghar, T. Naeem et al., "Antibacterial effect of mango (Mangifera indica Linn.) leaf extract against antibiotic sensitive and multi-drug resistant Salmonella typhi," Pakistan Journal of Pharmaceutical Sciences, vol. 26, no. 4, pp. 715-719, 2013.

[14] J. H. Doughari and M. Manzara, "In vitro antibacterial activity of crude leaf extracts of Mangifera indica Linn," African Journal of Microbiology Research, vol. 2, pp. 67-72, 2008.
[15] P. Jiamboonsri, P. Pithayanukul, R. Bavovada, and M. T. Chomnawang, "The inhibitory potential of Thai mango seed kernel extract against methicillin-resistant Staphylococcus aureus," Molecules, vol. 16, no. 8, pp. 6255-6270, 2011.

[16] S. U. Diso, M. Ali, S. I. Mukhtar, and M. Garba, "Antibacterial activity and phytochemical screening of mangifera indica (mango) stem and leaf extracts on clinical isolates of methicillin resistant Staphylococcus aureus," Journal of Advances in Medical and Pharmaceutical Sciences, vol. 13, pp. 2394-1111, 2017.

[17] I. Bairy, S. Reeja, Siddharth, P. S. Rao, M. Bhat, and P. G. Shivananda, "Evaluation of antibacterial activity of Mangifera indica on anaerobic dental microglora based on in vivo studies," Indian Journal of Pathology \& Microbiology, vol. 45, no. 3, pp. 307-310, 2002.

[18] P. R. Murray and E. J. Baron, Manual of Clinical Microbiology, ASM Press, Washington, DC, USA, 9th edition, 2007.

[19] M. Cheesbrough, Medical Laboratory Manual for Tropical Countries, Tropical Health Technology; Butterworths, London, UK, 2nd edition, 1987.

[20] NCCLS, Performance Standards for Antimicrobial Susceptibility Testing: Ninth Informational Supplement. M100-S11, National Committee for Clinical Laboratory Standards, Wayne, IL, USA, 2001.

[21] CLSI, Methods for Dilution Antimicrobial Susceptibility Tests for Bacteria that Grow Aerobically; Approved Standard-9th Edition, Document M07-A10, The Clinical and Laboratory Standards Institute (CLSI), Wayne, PA, USA, 2015.

[22] H. Uematsu, K. Yamashita, S. Kunisawa, K. Fushimi, and Y. Imanaka, "The economic burden of methicillin-resistant Staphylococcus aureus in community-onset pneumonia inpatients," American Journal of Infection Control, vol. 44, no. 12, pp. 1628-1633, 2016.

[23] A. F. Shorr, Y. P. Tabak, V. Gupta, R. Johannes, L. Z. Liu, and M. H. Kollef, "Morbidity and cost burden of methicillin-resistant Staphylococcus aureus in early onset ventilator-associated pneumonia," Critical Care, vol. 10, no. 3, p. R97, 2006.

[24] C.-C. Lai, K. Lee, Y. Xiao et al., "High burden of antimicrobial drug resistance in Asia," Journal of Global Antimicrobial Resistance, vol. 2, no. 3, pp. 141-147, 2014.

[25] M. E. Hamid, F. Y. Mustafa, A. Alwaily, S. Abdelrahman, and T. Al Azragi, "Prevalence of bacterial pathogens in aseer region, kingdom of Saudi Arabia: emphasis on antimicrobial susceptibility of Staphylococcus aureus," Oman Medical Journal, vol. 26, no. 5, pp. 368-369, 2011.

[26] C. C. Harish, "Antibacterial activity of Sphaeranthus indicus and Helianthus annus against multi-drug resistant (MDR) clinical isolates," International Journal of Advanced and Applied Sciences, vol. 3, no. 1, pp. 1-8, 2016.

[27] R. Thiyagarajan, Gunapadam Thathu Jeeva Vaguppu, Directorate of Indian Medicine and Hoemopathy, Chennai, India, 6th edition, 2006.

[28] S. Yezli, A. M. Shibl, D. M. Livermore, and Z. A. Memish, "Antimicrobial resistance among Gram-positive pathogens in Saudi Arabia," Journal of Chemotherapy, vol. 24, no. 3, pp. 125-136, 2012.

[29] M. E. S. Mirghani, F. Yosuf, N. A. kabbashi, J. Vejayan, and Z. B. M. Yosuf, "Antibacterial activity of mango kernel extracts," Journal of Applied Sciences, vol. 9, no. 17, pp. 3013-3019, 2009.

[30] A. K. Thabit, J. L. Crandon, and D. P. Nicolau, "Pharmacodynamic and pharmacokinetic profiling of delafloxacin in a murine lung model against community-acquired respiratory tract pathogens," International Journal of Antimicrobial Agents, vol. 48, no. 5, pp. 535-541, 2016. 